vom Wirte gebildeten Antikörper näher zu prüfen. Hierüber gedenken wir in einer spätern Mitteilung $z u$ berichten.

Ernst Gäumann und Otto JaAG

Institut für spezielle Botanik der Eidg. Technischen Hochschule, Zürich, den 5. März 1945.

\section{Beitrag zur Frage nach der Beteiligung lebender Stammzellen am Saftsteigen}

Da die Frage nach der Beteligung lebender Stammzellen am Saftsteigen noch nicht in allgemein anerkannter Weise beantwortet ist, sollten die physikalischen und physiologischen Vorgänge, die sich beim Abtöten durch Erwärmung abspielen, genauer analysiert werden.

Dic partielle Erwärmung des Stengels wurde in einem geeigneten Wasserbad vorgenommen; die Messung der Absorption und Transpiration erfolgte mit Potometer und Waage.

Zum Studium der physikalischen Prozesse dienten Versuche mit leblosen Modellen, besonders mit toten $Z$ weigstücken von Abies. Die Transpiration war hier ersetzt durch eine konstante Pumpensaugung. Die Bewegung des Meniskus in der Potometerkapillare stellt eine Resultante dar, die vornehmlich von zwei Komponenten abhängt: der Pumpensaugung, die den Meniskus vorwärts zu bewegen sucht und der Ausdehnung des sich erwärmenden Tracheideninhaltes, die den Meniskus rïckwärts $z u$ verschieben strebt. Je nach der Stärke der Saugung und der Art der Erwärmung läßt sich die Absorptionsgeschwindigkeit von positiven Werten über Null $z u$ negativen Werten überführen. In Betracht fällt ferner der Einflu $B$ der Temperatur auf die Viskosität des Wassers und auf die Permeabilität der Tracheidenwände.

Das Studium lebender Objekte erstreckt sich auf Koniferen, Mono- und Dikotylen. Es wurde die Absorption bei Vorhandensein und Fehlen der Transpiration untersucht.

Bei Unterdrückung der Transpiration durch Eintauchen der Sprosse unter Wasser, kann die Absorption noch stunden- und tagelang fortdauern. Es ist dies eine Folge der Saugkraft der lebenden Zellen und des Unterdruckes in den toten Leitungsbahnen. Bei der partiellen Erwärmung des Stengels ist zu unterscheiden das Verhalten unterhalb, innerhalb und oberhalb der praemortalen Zone. Unterhalb dieser Zone hat die Erwärmung im wesentlichen denselben EinfluB wie am toten Objekt. Innerhalb der praemortalen Zone steigt die Absorptionsgeschwindigkeit in charakteristischer Weise stark an, was nach eingehender Diskussion der Permeabilitätszunahme des lebenden Protoplasmas für Wasser zugeschrieben wird. In der letalen Zone preßt der Wanddruck aus den abgetöteten Zellen Saft aus; die Absorptionsgeschwindigkeit fällt dabei rasch ab und strebt bei Temperaturkonstan $z$ dem Nullwert zu.

Auf transpirierende Pflanzen wirkt die Erwärmung in der Hauptsache in gleicher Weise; doch geht hier die Absorption beim letalen Fallen nicht dem Nullwert entgegen, sondern sie steigt, der weiterdauernden Transpiration entsprechend, wieder an. Sie vermag aber die fortschreitende Unterbilanz nicht aufzuhalten, so da $B$ bald Welken und Verdorren eintritt. Die Erforschung der Ursachen dieser starken Unterbilanz ist von besonderem Interesse, da sie zur Frage nach der Beteiligung lebender Stammzellen beim Saftsteigen in Beziehung steht.
$\mathrm{Zu}$ diesem $\mathrm{Z}$ weck muBte auch die Transpiration untersucht werden. Es wurden Absorption und Transpiration an derselben Versuchspflanze gleichzeitig gemessen. Es zeigte sich, daB nach dem partiellen Abtöten des Stengels beide Größen sofort sinken, die Absorption aber bedeutend stärker als die Transpiration. Dadurch entsteht eine Unterbilanz, die zum Welken führt. Wohl ist auch bei der intakten Kontrollpflanze die Wasserbilanz am Tage gewöhnlich negativ, aber während der Nacht findet wieder eine ausreichende Erholung statt. Bei der Versuchspflanze dagegen wird die Unterbilanz so groB, da $B$ das Absterben nicht mehr $z u$ verhindern ist. Das Fallen der Absorption ist als Folge des Zellsaftaustrittes und der Wasseranreicherung der Leitungsbahnen physikalisch leicht verständlich; bei der Herabsetzung derTranspiration ist dies nicht der Fall. Um die Erklärungsmöglichkeiten für die schon nach $1 / 4$ Stunde deutliche Unterbilanz zu prüfen, war vor allem das Verhalten des Filtrationswiderstandes festzustellen.

Die Methode bestand in der Beobachtung der Filtration von Wasser unter konstantem Druck durch ein Stengelstück während und nach seiner partiellen $A b$ tötung. Eine durch das Abtöten bedingte Zunahme des Leitungswiderstandes konnte während der Beobachtungszeit (1-7 Tage) nicht nachgewiesen werden. Vergleiche mit den Änderungen der Wasserbilanz, die erst für wenige Spezies (Fuchsia, Plectranthus) vorliegen, führten zum Resultat, daß die Blätter $z u$ einer Zeit welken, wo eine Erhöhung des Leitungswiderstandes nicht nachzuweisen ist. Es sind also keine Unterlagen vorhanden, weder für irgendwelche Verstopfungen an den Grenzen lebend-tot, noch für jene hypothetischen Änderungen des Gefäßinhaltes oder der Gefäßwand, die den Fil trationswiderstand steigern sollen. Soweit die derzeitigen Erfahrungen reichen, besteht die plausibelste Erklärung für das Welken nach partiellem Abtöten in der Annahme von Hebungskräften im Stengel, die durch das Abtöten zerstört werden.

A. URSPRUNG und J. KOLLER

Botanisches Institut, Fribourg, den 6. März 1945.

\section{La culture d'Eremothecium Ashbyii en milieu synthétique}

Le rôle de certains acides aminés

E. Ashbyil (Ascomycète) possède un pouvoir de synthèse remarquable pour la lactoflavine. Cette dernière s'accumule dans le milieu et peut également cristalliser dans les vacuoles des hyphes (GUILLERMond ${ }^{1}$ ).

Ce microorganisme nous permet d'étudier le déterminisme de la flavinogenèse, à condition qu'on puisse le cultiver sur un milieu synthétique. Sur un milieu naturel à base de peptone, il se développe bien en produisant des quantités de flavine dépassant $1 \mathrm{mg}$. pour $25 \mathrm{ccm}$. de milieu. Cependant, sur un milieu synthétique à base de glycocolle, de glucose et de sels minéraux, aucune croissance ne se produit. L'adjonction de biotine, d'aneurine et de mésoinositol, qui sont pourtant présents dans la peptone, est inefficace.

Les faits suivants ont déjà été établis, (SCHOPFER2). La peptone traitée par la norite devient inactive. Une

A A. Gullermond, Rev, Mycologie, 1, 115, (1936),

a W. H. Schopfer, Helv, Chim. Acta, 27. 1017 (1943). 